\title{
ELECTRON-HOLE ASYMMETRY: THE KEY TO SUPERCONDUCTIVITY
}

\author{
J.E. Hirsch \\ Department of Physics \\ University of California, San Diego, La Jolla, CA 92093
}

The observation that superconductors have predominantly positive Hall coefficients was made already in 1962 by Chapnik. The discovery of superconductivity in hole-doped oxides in 1986, and the measurement of a positive Hall coefficient in electron-doped oxides in 1989, further underscore the existence of a distinct asymmetry between electrons and holes with respect to superconductivity, which calls for an explanation. No such explanation is provided by most proposed superconductivity mechanisms including the electron-phonon interaction. Here we discuss the simplest possible local interaction that breaks electron-hole symmetry in solids. This interaction causes holes to be heavier than electrons, and is repulsive for electrons and attractive for holes. Thus, it provides an explanation for the above observations. It leads to superconductivity in the absence of any other attraction mechanism, and the resulting theory exhibits many characteristic features observed in the high $T_{c}$ oxides. We review the predictions of the theory and their relationship with existing and future experiments in high $T_{c}$ oxides. A brief discussion of the implications of this mechanism for the understanding of superconductivity in "conventional materials" is also given.

\section{CHAPNIK'S RULE}

Empirical rules play an important role in the development of science. Whether or not they directly guide towards the correct theory, when a consistent theory finally emerges its agreement with empirical rules can immediately lend it strong support, especially if the theory was not specifically devised to account for those rules. To draw an example from the area of molecular biology (following a leading theorist in our field), the fact that Watson and Crick's base pairing model naturally explained Chargaff's rules on the proportion of the different bases in DNA strands immediately lent strong support to that model. In the field of superconductivity, in contrast, empirical rules (such as those used by B. Matthias and others to find new superconducting compounds) and theory have usually been strangely at odds. One may wonder why.

Here I would like to focus on one such rule, that was formulated almost 30 years ago by I.M. Chapnik, 1 hence I will call Chapnik's rule: "the existence of holes is important for the occurrence of superconductivity". Chapnik observed that among the elements essentially all the superconductors have positive Hall coefficient, and that non-superconductors like the simple and noble metals have negative Hall coefficient (note that even $\mathrm{Al}$ has a positive Hall coefficient at high fields). ${ }^{2}$ Chapnik later extended his observation to various compounds and suggested that it would be profitable to systematically study the Hall coefficient in a variety of superconducting compounds where it has not been measured. In essentially all known cases the low temperature, high field Hall coefficient being negative in all directions implies absence of superconductivity. 
Although some experimentalists were probably aware of this rule in the past and guided by it in the search for new superconducting compounds, 3 it did not come to the attention of theorists, and there has been to my knowledge not a single attempt to provide theoretical justification for it. Perhaps for this reason Chapnik's rule was unknown to the vast majority of the current generation of physicists (including myself), and when the high $\mathrm{T}_{c}$ oxides were discovered in 1986 it did not become immediately obvious that they were a prime example of this rule: they became superconducting when doped with holes, exhibiting a positive Hall coefficient, and superconductivity disappeared on further doping when the Hall coefficient changed sign. 4

The situation became less clear-cut again in January 1989, when the discovery of "a superconducting copper-oxide compound with electrons as the charge carriers" was announced, 5 in apparent violation of Chapnik's rule. Fortunately, by that time the theory to be discussed here was already sufficiently developed that we were able to immediately predict that in this case "theory would prove experiments wrong" and that hole carriers would be found in these materials when more careful transport and spectroscopic measurements were performed. 6 It was also suggested how $\mathrm{O}$ holes would naturally be induced by electron doping of these materials due to the absence of apex oxygens in the structures. 6,7 The evidence that hole carriers indeed exist in these materials is mounting 8,9 and in particular Hall coefficient measurements on single crystals are finding positive values for magnetic field perpendicular to the planes, as predicted.

The conventional electron-phonon mechanism of superconductivity is manifestly particle-hole symmetric, and there is no element in the theory that would suggest that the sign of the curvature of the Fermi surface could make a difference in the magnitude of the coupling constants that enter the theory. Nor do most other mechanisms for superconductivity proposed in the past as well as for the new materials, such as excitonic-,plasmon-,magnon- or anharmonic phonon-mediated pairing, make a distinction between electrons and holes. In view of the vast empirical evidence in favor of Chapnik's rule for the old as well as the new materials we believe it is imperative for these theories to address the issue and suggest an explanation within their framework. Instead, electron-hole asymmetry is the cornerstone of the theory to be discussed in this paper, which in fact leads to what I would term a "strong form" of Chapnik's rule: all superconductors are hole superconductors. By that I mean that it is the pairing of hole carriers that drives superconductivity, in all cases.

\section{THE BASIC PRINCIPLE: HOLES ARE HEAVIER THAN ELECTRONS}

The essence of high $T_{c}$ is, I believe, clearly displayed in the plot by Torrance and coworkers, ${ }^{10}$ reproduced from their paper in Fig. 1. The qualitative behavior of $T_{c}$ versus hole concentration shown here has since been found by many other workers in a wide range of samples and materials. The Figure also indicates that states are "localized" for small hole doping and the system is a "normal metal" for large hole doping. This refers to the rapid decrease in the normal state resistivity that occurs upon hole doping, that is observed in all the oxide superconductors.

The way to read Fig. 1 that leads directly to the theory under discussion here is as follows: a few holes added to the insulator have difficulty propagating as single objects, hence they are almost localized and the normal-state resistivity is very high. In this regime the holes pair as the temperature is lowered and the system becomes a superconductor (the initial rise in $\mathrm{T}_{c}$ versus hole doping is simply related to the increasing number of carriers.) When enough holes have been added to the system it becomes a "normal metal" with a large mobility for holes in the normal state, and in this regime holes don't pair and don't become superconducting. The conclusion that holes pair because they have difficulty propagating individually, in order to increase their ability to propagate, and cease to pair when their ability to propagate individually has become large, is almost inescapable. In fact, it can be derived in a phenomenological way from the single assumption that the hole mobility is an increasing function of hole concentration, which can be "read off" from measurements of resistivity versus doping.

We assume that the increase in hole mobility with doping arises from a decrease in the hole's effective mass, or equivalently an increase in the hopping amplitude $t$ between nearest neighbor sites. To lowest order in the hole density $n_{h}$ we have then for the hopping amplitude $t$ as function of hole concentration: 


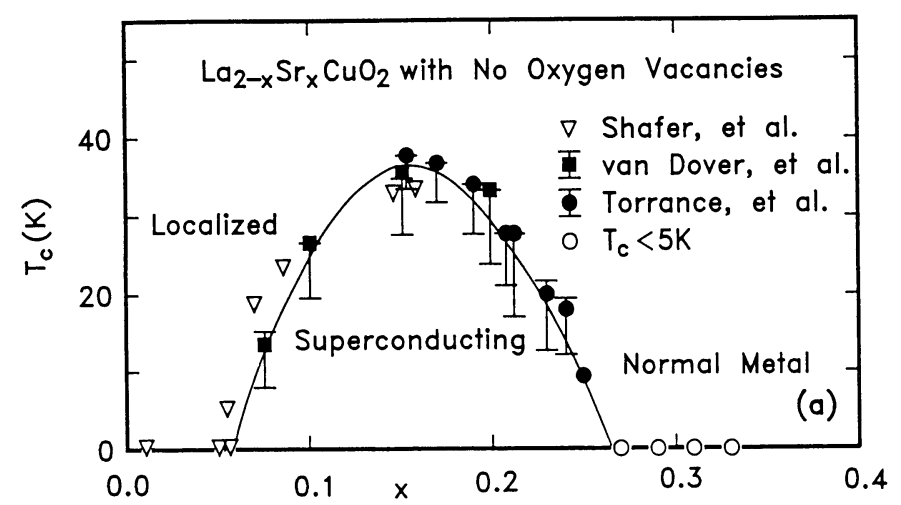

Fig. 1. $\mathrm{T}_{\mathrm{c}}$ versus $\mathrm{Sr}$ content in $\mathrm{La}_{2-\mathrm{x}} \mathrm{Sr}_{\mathrm{x}} \mathrm{CuO}_{4}$, from Ref. 10. $\mathrm{x} / 2$ is expected to correspond to number of holes added per $\mathrm{O}$ atom in the planes.

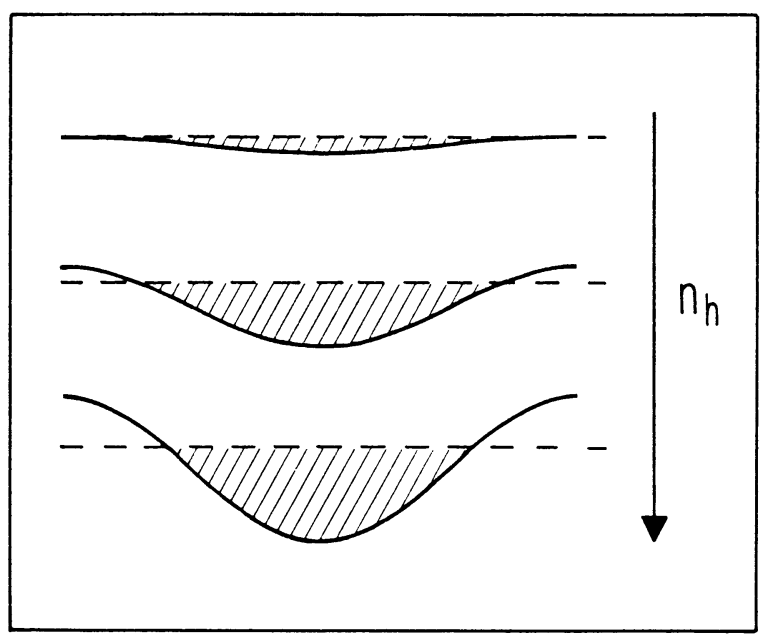

Fig. 2. The relevant electronic band for various hole contents (schematic). We expect this band to arise from overlap of $\mathrm{O} \mathrm{p} \pi$ orbitals in the planes.

$$
t\left(n_{h}\right)=t_{h}+\Delta t n_{h},
$$

with

$$
\Delta \mathrm{t}=\frac{\partial \mathrm{t}}{\partial \mathrm{n}_{\mathrm{h}}}>0 .
$$

The fact that for a few holes in the band the states are localized indicates that $t_{h}$ in the oxides is close to zero, and the observed bandwidth at finite doping originates mainly in the $\Delta t$ term in Eq. (1). Fig. 2 shows schematically the single-particle band for different values of hole doping. 
Now it is reasonable to assume that the density dependance of the hopping amplitude arises from the local hole density dominantly, i.e. the hole density in the two sites involved in the hopping process. For a hole of spin $\sigma$ hopping between sites $i$ and $j$ the exclusion principle prevents another hole of the same spin from being at those sites so that the hopping amplitude $t_{i j}$ depends on the occupation of those sites by holes of opposite spin:

$$
\mathrm{t}_{\mathrm{ij}}=\mathrm{t}_{\mathrm{h}}+\Delta \mathrm{t}\left(\mathrm{n}_{\mathrm{i},-\sigma}+\mathrm{n}_{\mathrm{j},-\sigma}\right)
$$

The kinetic energy for holes is then given by

$$
H_{k i n}=-\sum_{<i j>, \sigma} t_{i j}\left(c_{i \sigma}^{+} c_{j \sigma}+\text { h.c. }\right)=-\sum_{<i j>, \sigma}\left(t_{h}+\Delta t\left(n_{i,-\sigma}+n_{j,-\sigma}\right)\right)\left(c_{i \sigma}^{+} c_{j \sigma}+\text { h.c. }\right)
$$

and thus the density dependance of the hopping naturally gives rise to an interaction term in the Hamiltonian from the last term in Eq. (4):

$$
V_{h}=-\Delta t \sum_{<i j>, \sigma}\left(n_{i,-\sigma}+n_{j,-\sigma}\right)\left(c_{i \sigma}^{+} c_{j \sigma}+\text { h.c. }\right) \text {. }
$$

This "hopping interaction" is most attractive for a few holes in a filled band, it becomes smaller as more holes are added and is repulsive when the band is more than half-full with holes (less than half-full with electrons). It gives rise to superconductivity with a $\mathrm{T}_{\mathrm{c}}$ versus concentration dependance of the form shown in Fig. 1, as discussed in later sections. We have proposed that this interaction is responsible for superconductivity in all solids. 11

In principle one could imagine various different origins for the physics discussed above, leading to the same phenomenological Hamiltonian. For example, it has been pointed out that a single hole in an antiferromagnetic background cannot propagate easily because it leaves behind a "string" of overturned spins, while two holes can propagate together without disrupting the background.12 This point of view has been independently emphasized in a recent series of papers by Izuyama 13, and it is also a simple way to understand pairing by fractional statistics as advocated by Laughlin. 14 It leads naturally to a picture of heavy single holes that pair to increase their mobility, that could be described "phenomenologically" by a kinetic energy of the form Eq. (4). In such a picture pairing would occur in doping a half-filled band antiferromagnetic insulator in either direction (i.e. with electrons or holes), which would be in apparent agreement with some observations. However, we emphasize that our point of view here is very different.

The Hamiltonian Eq. (4) leads to pairing with arbitrarily short coherence length depending on the relative sizes of $t_{h}$ and $\Delta t$. In fact, for $t_{h}=0$ two holes will propagate only if they are on the same or nearest neighbor sites, so that the coherence length is less than a single lattice spacing. It is interesting to contrast the physics described by Eq. (4) with the case of the attractive Hubbard model:

$$
H=-t \sum_{<i j>, \sigma}\left(c_{i \sigma}^{+} c_{j \sigma}+\text { h.c. }\right)+U \sum_{i} n_{i \uparrow} n_{i \downarrow}
$$

with $U<0$. This model is widely used as a paradigm for short coherence length superconductors, and can be thought of also as an "effective model" describing more general forms of densitydensity attractive interactions. Here, short coherence length also occurs for small $t$ (or large $\mathrm{U})$, but in this regime the mobility of the pairs becomes very small, since the pair effective hopping is

$$
\mathrm{t}_{\mathrm{eff}}=\mathrm{t}^{2} /|\mathrm{U}|
$$

In other words, the pair interaction limits the mobility, in contrast to the interaction in Eq. (4) that enhances the mobility. The fact that the ultimate result of pairing is to superconduct, that is an enhanced ability for the carriers to propagate, favors a Hamiltonian of the form Eq. (4) rather than Eq. (6). 
Beyond these "philosophical" remarks, there is a concrete difference between the physics described by Eq. (4) and Eq. (6) in the regime of short coherence length (relevant to the high $\mathrm{T}_{\mathrm{c}}$ oxides). In the attractive Hubbard model as the coherence length becomes shorter the pair binding energy $E_{b}(U)$ increases relative to the effective hopping Eq. (7):

$$
\mathrm{E}_{\mathrm{b}} \gg \mathrm{t}_{\mathrm{eff}}
$$

and because of this the transition temperature for Bose condensation is much lower than for pair formation. Thus, the superconducting state is destroyed by center of mass excitations of the pairs rather than pair dissociation, and pairs still exist above $\mathrm{T}_{\mathrm{c}}$. In contrast, in the case described by Eq. (4) the effective hopping for a pair remains finite as the coherence length is reduced and in the presence of Coulomb repulsion the pair binding energy is usually much smaller than the pair hopping amplitude: 15

$$
\mathrm{E}_{\mathrm{b}}<<\mathrm{t}_{\mathrm{eff}}
$$

As a consequence, the finite temperature transition is a BCS pair-unbinding transition even in the regime of very short coherence length. The absence of pairs above $T_{c}$ in the oxide superconductors inferred from NMR experiments 16 thus supports the physics described by Eq. (4) rather than by Eq. (6). In fact we believe that the combined observations of short coherence length and absence of pairs above $\mathrm{T}_{\mathrm{c}}$ cannot be described by a Hamiltonian involving only density-density interactions (as in Eq. (6) and generalizations) and imply a kinematic pairing mechanism as described by Eq. (4).

\section{MICROSCOPIC ORIGIN OF $\triangle \mathrm{t}$ INTERACTION}

Within the framework of a single-band tight binding model the interaction $\Delta t$ is simply the "hybrid" matrix element of the interaction between quasiparticles: 11

$$
\Delta t=\int d^{3} r^{3} r^{\prime}\left|\varphi_{i}(r)\right|^{2} V_{e e}\left(r-r^{\prime}\right) \varphi_{i}^{*}\left(r^{\prime}\right) \varphi_{j}\left(r^{\prime}\right)
$$

with $V_{\text {ee }}\left(r-r^{\prime}\right)$ the effective (repulsive) interaction between electrons at positions $r$, $r^{\prime}$. The wavefunctions $\varphi_{i}, \varphi_{j}$ represent atomic orbitals or Wannier functions at sites $i$ and $j$. The single particle hopping matrix element is given by

$$
-t_{0}=\int d^{3} r V_{e i}\left(r-R_{j}\right) \varphi_{i}^{*}(r) \varphi_{j}(r)
$$

with $V_{e i}$ the (attractive) potential for an electron due to the ion at lattice site $R_{\mathrm{j}}$. Because of the opposite signs of $\mathrm{V}_{\mathrm{ee}}$ and $\mathrm{V}_{\mathrm{ei}}$ the signs of $\left(-\mathrm{t}_{0}\right)$ and $\Delta \mathrm{t}$ are always opposite. This implies that the interaction generated by $\Delta t$ is always attractive (repulsive) for quasiparticle states at the top (bottom) of the electronic energy bands. In other words, the constraint that states at the top of the band have to be orthogonal to those below them ensures that the interaction Eq. (10a) is most attractive for the band states with highest quasiparticle energies. With the convention that the phase of the wavefunctions $\varphi_{\mathrm{i}}, \varphi_{\mathrm{j}}$ is the same in the region between sites $\mathrm{i}$ and $\mathrm{j}, \Delta \mathrm{t}$ and $\mathrm{t}_{0}$ are positive.

However, this is only part of the story. An important contribution to $\Delta t$ arises from the modification of the electronic wavefunction in an atom by the presence of other electrons. This effect was described by a phenomenological model with a pseudospin degree of freedom, 17 and more recently with a tight binding model with two orbitals per site. 18 These models lead to a reduced hopping rate for holes as compared to electrons due to the larger modification of the "background" to the hole when it hops from site to site. In the tight binding model the physics is depicted in Fig. 3. We assume two atomic orbitals, $s$ and s', and Coulomb repulsions such that two electrons go into the higher single-particle level. The hopping amplitude is smaller when 
there are more electrons (fewer holes) due to the overlap matrix element between initial and final states.

Both of the effects discussed above are of the same sign and we believe it is a combination of these that leads to values of $\Delta t$ of the magnitude necessary to give rise to superconductivity. In fact, without taking into account the modification of atomic states by the presence of other electrons the value of $\Delta t$ obtained from Eq. (10a) is close to zero when orbitals that are orthogonal at nearest neighbor sites are used.19 Thus it is fundamentally the disruption that a hole causes in its environment when it propagates that limits its ability to do so, while a single electron outside closed shells can propagate essentially unimpeded. In this respect the charge state of the ions also plays an important role: the largest $\Delta t$ will occur when in the filled band the

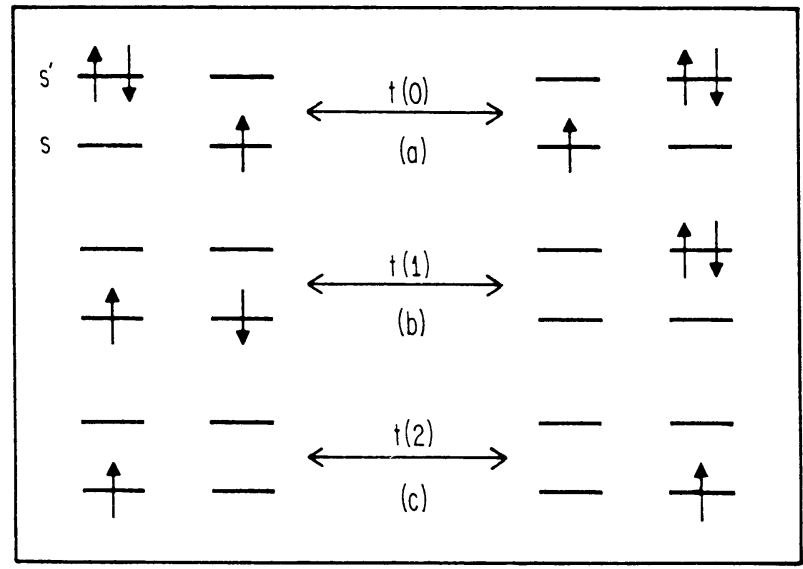

Fig. 3. Electrons (arrows) hopping between neighboring sites in the tight binding model of Ref. 18, with 2 orbitals per site. As the number of electrons (holes) increases (decreases) the hopping amplitude decreases: $t(2)>>t(1)>>t(0)$. $\Delta \mathrm{t}=\mathrm{t}(1)-\mathrm{t}(0)$.

ion is most unstable, i.e. for holes propagating through negatively charged anions. In such a situation a hole will cause a large change in the state of the anion. 17 This is the case in the oxide superconductors where our picture suggests that the carriers that give rise to superconductivity are $\mathrm{p} \pi$ holes conducting by direct hopping through $\mathrm{O}=$ anions.

There is a direct "empirical" way to determine the magnitude of $\Delta t$ in a diatomic molecule, by the difference in excitation energies of the molecule with different number of electrons. As the simplest possible example, discussed in Ref. 20, the difference in excitation energies of $\mathrm{H}_{2}{ }^{+}$ and $\mathrm{H}_{2}$ - gives a direct measure of the interaction $\Delta t$ in that case. More generally through spectroscopic analysis one can obtain information on the magnitude of $\Delta \mathrm{t}$ by fitting optical spectra, and in fact such a term was "discovered" experimentally by Zaanen et al. in attempting to interpret spectroscopic data in Ni fluorides. 21 The configuration dependance of hopping matrix elements in tight binding models has also been recently emphasized by Gunnarson et al.22 Zawadowski 23 has also recently considered the effect of such terms on superconductivity in a two-band model. 


\section{THE THEORY}

The theory of hole superconductivity based on the interaction Eq. (5) was developed by F. Marsiglio and the author. I will summarize some of the main aspects here and in the next section, and the reader is referred to the references $(24-27,11,15)$ for additional information.

We start from the single band Hamiltonian:

$$
H=-\sum_{<i j>, \sigma} t_{i j}\left(c_{i \sigma}^{+} c_{j \sigma}+\text { h.c. }\right)+\sum_{<i j>, \sigma}(\Delta t)_{i j}\left(c_{i \sigma}^{+} c_{j \sigma}+\text { h.c. }\right)\left(n_{i,-\sigma}+n_{j,-\sigma}\right)+\sum_{<i j>} v_{i j} n_{i} n_{j}(11)
$$

and assume that the quantities $\mathrm{t}_{\mathrm{ij}}$ and $(\Delta \mathrm{t})_{\mathrm{ij}}$ are proportional:

$$
(\Delta t)_{\mathrm{ij}} / t_{\mathrm{ij}}=\alpha
$$

which is a reasonable assumption since both originate in the same overlap matrix element, as seen in Eq. (10). Here, $\mathrm{c}_{\mathrm{i} \sigma}^{+}$creates an electron of spin $\sigma$ at site $\mathrm{i}$ (in contrast to Eq. (4) where $\mathrm{c}_{\mathrm{i} \sigma}^{+}$is a hole creation operator). In Fourier space, Eq. (11) is:

$$
\mathrm{H}=\sum_{\mathrm{k}, \sigma} \varepsilon_{\mathrm{k}} \mathrm{c}_{\mathrm{k} \sigma}^{+} \mathrm{c}_{\mathrm{k} \sigma}+\sum_{\mathrm{k}, \mathrm{k}^{\prime}, \sigma, \sigma^{\prime}}\left[(-\alpha)\left(\varepsilon_{\mathrm{k}}+\varepsilon_{\mathrm{k}^{\prime}}+\varepsilon_{\mathrm{k}+\mathrm{q}}+\varepsilon_{\mathrm{k}^{\prime}-\mathrm{q}}\right)+\mathrm{V}(\mathrm{q})\right] \mathrm{c}_{\mathrm{k}+\mathrm{q} \sigma}^{+} \mathrm{c}_{\mathrm{k}^{\prime}-\mathrm{q} \sigma^{\prime}}^{+} \mathrm{c}_{\mathrm{k}^{\prime} \sigma^{\prime}} \mathrm{c}_{\mathrm{k} \sigma}
$$

with

$$
\begin{aligned}
& \varepsilon_{k}=\sum_{j} e^{-i k R_{j}} t_{0 j} \\
& V(q)=\sum_{j} e^{-i q R_{j}} V_{0 j}
\end{aligned}
$$

The essential feature of the second term in Eq. (13) is that the interaction depends not only on momentum transfer $q$ as is usually the case but also on the incoming momenta $\mathrm{k}$ and k'. Such an interaction cannot exist in a free electron system where the wave functions are plane waves. 11 Thus, the discreteness imposed by the lattice of ions is absolutely essential to the phenomenon of superconductivity.

The pair interaction for scattering from $(\mathrm{k},-\mathrm{k})$ to $\left(\mathrm{k}^{\prime},-\mathrm{k}^{\prime}\right)$ is given by

$$
\mathrm{V}_{\mathrm{kk}^{\prime}}=-2 \alpha\left(\varepsilon_{\mathrm{k}}+\varepsilon_{\mathrm{k}^{\prime}}\right)+\mathrm{V}\left(\mathrm{k}-\mathrm{k}^{\prime}\right)
$$

and it is clearly least repulsive for largest $\varepsilon_{\mathrm{k}}, \varepsilon_{\mathrm{k}^{\prime}}$, i.e. at the top of the band. To conform to our notation in previous papers we do a particle-hole transformation and obtain

$$
\mathrm{V}_{\mathrm{kk}}=2 \alpha\left(\varepsilon_{\mathrm{k}}+\varepsilon_{\mathrm{k}^{\prime}}\right)+\mathrm{V}\left(\mathrm{k}-\mathrm{k}^{\prime}\right)
$$

describing now the scattering of hole quasiparticles of energy $\varepsilon_{\mathrm{k}}, \varepsilon_{\mathrm{k}^{\prime}}$. Although in principle the problem could be solved for arbitrary $\mathrm{V}\left(\mathrm{k}-\mathrm{k}^{\prime}\right)$ it becomes enormously simpler if we have to deal only with energy variables rather than momenta. This is the case if we only keep on-site and nearest-neighbor Coulomb repulsions, and also assume that the hopping involves only nearest neighbor sites. The pair interaction becomes:

$$
\mathrm{V}_{\mathrm{kk^{ \prime }}}=\mathrm{U}+2 \alpha\left(\varepsilon_{\mathrm{k}}+\varepsilon_{\mathrm{k}^{\prime}}\right)+\alpha^{\prime} \varepsilon_{\mathrm{k}} \varepsilon_{\mathrm{k}^{\prime}}
$$

where we have assumed 


$$
\alpha^{\prime}=V_{\mathrm{ij}} / t_{\mathrm{ij}}
$$

independent of direction, and $\mathrm{U}=\mathrm{V}_{\mathrm{ij}}$ for $\mathrm{i}=\mathrm{j}=0$ is the on-site repulsion. Here we have left out some terms arising from $\mathrm{V}\left(\mathrm{k}-\mathrm{k}^{\prime}\right)$ that are odd under $\mathrm{k} \rightarrow-\mathrm{k}$ or $\mathrm{k}^{\prime} \rightarrow-\mathrm{k}^{\prime}$ that drop out in the subsequent development. We should also point out that the origin of band energies is chosen such that

$$
\sum_{\mathrm{k}} \varepsilon_{\mathrm{k}}=0
$$

as follows from Eq. (14a) taking $t_{i j}=0$ for $i=j$.

The pair interaction Eq. (17) has a very simple form and in fact can be considered as having wider applicability than the conditions invoked above to derive it. It follows simply from the assumption that the dominant variation in the interaction arises from quasiparticle energy rather than momentum:

$$
\mathrm{V}_{\mathrm{kk}^{\prime}}=\mathrm{V}_{\mathrm{eff}}\left(\varepsilon_{\mathrm{k}}, \varepsilon_{\mathrm{k}^{\prime}}\right)
$$

assuming symmetry in the energy indices. Then,

$$
\begin{aligned}
& \mathrm{U}=\mathrm{V}_{\mathrm{eff}}(0,0) \\
& \left.\alpha=\frac{1}{2} \frac{\partial \mathrm{V}_{\mathrm{eff}}}{\partial \varepsilon_{\mathrm{k}}}\right)_{0,0} \\
& \left.\alpha^{\prime}=\frac{\partial^{2} \mathrm{~V}_{\mathrm{eff}}}{\partial \varepsilon_{\mathrm{k}} \partial \varepsilon_{\mathrm{k}^{\prime}}}\right)_{0,0}
\end{aligned}
$$

and higher order contributions are neglected. As will be seen below, the assumption Eq. (20) has the fundamental consequence that the gap is constant over the Fermi surface.

We use the usual BCS equation

$$
\Delta\left(\varepsilon_{\mathrm{k}}\right)=-\frac{1}{\mathrm{~N}} \sum_{\mathrm{k}^{\prime}} \mathrm{V}\left(\varepsilon_{\mathrm{k}}, \varepsilon_{\mathrm{k}^{\prime}}\right) \Delta\left(\varepsilon_{\mathrm{k}^{\prime}}\right) \frac{\tanh \left(\beta \frac{\mathrm{E}_{\mathrm{k}^{\prime}}}{2}\right)}{2 \mathrm{E}_{\mathrm{k}^{\prime}}}
$$

and the constraint condition for the density of holes

$$
\mathrm{n}=1-\frac{1}{\mathrm{~N}} \sum_{\mathrm{k}} \frac{\varepsilon_{\mathrm{k}}-\mu}{\mathrm{E}_{\mathrm{k}}} \tanh \left(\beta \frac{\mathrm{E}_{\mathrm{k}}}{2}\right)
$$

with the quasiparticle energy given by

$$
\mathrm{E}_{\mathrm{k}}=\sqrt{\left(\varepsilon_{\mathrm{k}}-\mu\right)^{2}+\Delta^{2}\left(\varepsilon_{\mathrm{k}}\right)}
$$

and $\mu$ the chemical potential. The sums in Eq. (22) can be transformed to integrals over energy using the single particle density of states. Eq. (22a) and the form of the potential Eq. (17) imply that the gap function $\Delta\left(\varepsilon_{\mathrm{k}}\right)$ has a linear dependance on band energy, which we parametrize as:

$$
\Delta\left(\varepsilon_{\mathrm{k}}\right)=\Delta_{\mathrm{m}}\left(-\frac{\varepsilon_{\mathrm{k}}}{\mathrm{D} / 2}+\mathrm{c}\right)
$$


where $\mathrm{D}$ is the single-particle bandwidth and the parameters $\Delta_{\mathrm{m}}$ and $\mathrm{c}$ are determined by solution of Eq. (22), as discussed in the references. The quasiparticle gap is obtained from minimization of Eq. (23) as:

$$
\begin{aligned}
& \Delta_{0}=\frac{\Delta(\mu)}{\mathrm{a}} \\
& \mathrm{a}=\left(1+\left(\frac{\Delta_{\mathrm{m}}}{\mathrm{D} / 2}\right)^{2}\right)^{1 / 2}
\end{aligned}
$$

and in terms of it the quasiparticle energy is

$$
\mathrm{E}_{\mathrm{k}}=\sqrt{\mathrm{a}^{2}\left(\varepsilon_{\mathrm{k}}-\varepsilon_{0}\right)^{2}+\Delta_{0}^{2}}
$$

with

$$
\varepsilon_{\mathrm{O}}=\mu+\frac{1}{\mathrm{a}} \frac{\Delta_{\mathrm{m}}}{\mathrm{D} / 2} \Delta_{\mathrm{O}}
$$

Note that a shift in the minimum of $E_{k}$ occurs due to the energy dependance of the gap.

A fundamental feature of the interaction Eq. (17) is that because it depends strongly on the position of the states in the band it can give rise to superconductivity even if it is repulsive throughout the band. 24 This is analogous to the "pseudopotential effect" in electron-phonon theory. 28 The gap function Eq. (24) will change sign in the region where $\mathrm{V}_{\mathrm{kk}}$ ' is most repulsive, leading to a solution of Eq. (22) even for $V_{k k}>0$ throughout the band. This is an appealing feature of the mechanism discussed here and is absent in other mechanisms for superconductivity proposed originating in non-retarded interactions. 29

A second important feature of this model is that its solution is given accurately by BCS theory, contrary to most other models 15,26 . This is because superconductivity only occurs for a dilute concentration of (hole) carriers. It is well-known that BCS theory accurately describes the superconducting ground state in dilute systems even in the regime of short coherence length, 30 and in the model discussed here the finite temperature transition is also described by BCS theory due to the fact that the pair binding energy is much smaller than its hopping rate, as discussed in the previous section.

Quantitative solution of the Eqs. discussed here is given in the references. It is found that very "plausible" values of the interactions in the model give rise to superconductivity with critical temperatures of the magnitude observed. The condition for occurrence of superconductivity in the limit of small hole concentration is

$$
\frac{\Delta \mathrm{t}}{\mathrm{t}_{\mathrm{h}}}>\sqrt{\left(1+\frac{\mathrm{U}}{2 \mathrm{z} \mathrm{t}_{\mathrm{h}}}\right)\left(1+\frac{\mathrm{V}}{2 \mathrm{t}_{\mathrm{h}}}\right)}-1
$$

with $\mathrm{U}$ and $\mathrm{V}$ the on-site and nearest-neighbor Coulomb repulsion, $\Delta \mathrm{t}$ the hopping interaction Eq. (5), $\mathrm{z}$ the number of nearest neighbors and $\mathrm{t}_{\mathrm{h}}$ the single hole hopping amplitude at the top of the band

$$
\mathrm{t}_{\mathrm{h}}=\mathrm{t}_{0}-2 \Delta \mathrm{t}
$$

with $t_{0}$ the hopping amplitude at the bottom of the electron band, Eq. (10b). In the limit $t_{h} \rightarrow 0$ (for $\Delta t=t_{0} / 2$ ) the criterion Eq. (28) reduces to the simple form:

$$
\Delta \mathrm{t}>\sqrt{\frac{\mathrm{UV}}{4 \mathrm{z}}} .
$$


Analytic forms for the critical temperature in weak and strong coupling regimes are given in the references. The weak coupling $\mathrm{T}_{c}$ equation has been independently derived by Micnas et al.31 In the next section we summarize the main results of the theory for a variety of observables.

\section{CONSEQUENCES AND COMPARISON WITH EXPERIMENT}

We summarize here the main consequences of the theory and its relationship to existing and future experiments:

\section{(1) Doping dependance of $T_{c}$}

Because of the energy dependance of the interaction Eq. (17) superconductivity is restricted to low hole density. Physically, the hopping interaction becomes increasingly less attractive as holes are added to a filled band. $T_{c}$ versus $n_{h}$ exhibits the typical behavior shown in Fig. 4. We have included the effect of the single particle hopping renormalization Eq. (1). As discussed in Ref. 25 within a single band model there is not much freedom in adjusting the range of hole concentration where $T_{c}$ is non-zero which is typically in the range of 0.15 to 0.2 holes per 0 atom, in agreement with observations (note that the hole concentration given in Fig. 1 is per $\mathrm{Cu}$ ion in the plane). The behavior shown in Fig. 1 has been reproduced in a variety of samples and little doubt remains that it is intrinsic.

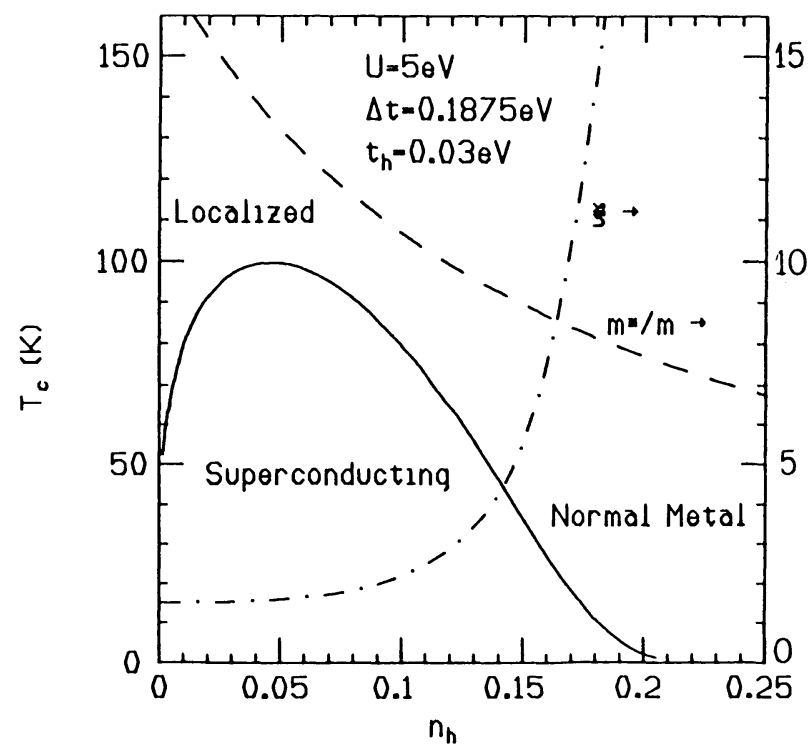

Fig. 4. $\mathrm{T}_{\mathrm{c}}$ versus hole concentration $\mathrm{n}_{\mathrm{h}}$ in the single band model for a representative set of parameters. The behavior of the coherence length $\xi$ (as defined in Ref. 26) and effective mass versus $n_{h}$ is also shown.

(2) Gap isotropy

The gap Eq. (24) is constant over constant energy surfaces even for anisotropic band structures. This followed from the fact that the hopping interaction has the same distance dependance as the single particle hopping (Eq. (12)) together with the assumption Eq. (18). In fact, some deviations from this can occur in an anisotropic material due to deviations from Eq. (18), i.e. the Coulomb repulsion will in general 
have a different distance dependance. However, the anisotropy introduced by this effect turns out to be only a few percent even in very anisotropic structures, as shown in Ref. 25. There is evidence from photoemission experiments that the gap is isotropic in the plane, 32 but no information yet on this question in directions off the plane. Observation of a large anisotropy would be inconsistent with the theory.

\section{(3) Pressure dependance of $\underline{T}_{\mathcal{c}}$}

Because the hopping interaction $\Delta \mathrm{t}$ increases when the atoms are closer together, pressure will in general increase $\mathrm{T}_{\mathrm{c}}$ in this model. More specifically however, in an anisotropic material $\mathrm{T}_{\mathrm{c}}$ will increase rapidly with pressure in the planes and is only weakly dependent on pressure perpendicular to the planes. Quantitative results are given in Ref. 25. With increasing doping the model predicts the pressure derivative to increase.

Initial reports suggested that pressure perpendicular to the planes has a similar effect to hydrostatic pressure, 33 in contradiction to the theory. However, recent measurements of thermal expansion coefficients indicate an anisotropic pressure dependance 34 as predicted by the theory, as do measurements of critical temperature in strained multilayer films. 35 More extensive experiments on this question are highly desirable, as well as measurements of the change in pressure dependance of $\mathrm{T}_{\mathrm{c}}$ with doping. The theory also predicts a larger in-plane stiffness for electrondoped compared to hole-doped materials ${ }^{36}$ which should be experimentally verifiable.

\section{(4) Tunneling asymmetry}

Because of the energy dependance of the gap the tunneling density of states exhibits an asymmetry of universal sign: the tunneling current in an N-I-S junction should be larger for a negatively-biased sample. Quantitative estimates are given in reference 27. Although many tunneling experiments appear to show an asymmetry of the sign predicted the evidence is not yet conclusive. It would be desirable that the polarity convention be clearly stated in reports of tunneling experiments.

\section{(5) Strong to weak coupling cross-over}

Because of the single particle hopping density dependance Eq. (1) as well as the energy dependance of the interaction Eq. (17) a cross-over from strong to weak coupling occurs as the hole concentration increases. In particular the normal state effective mass and the superconducting coherence length are predicted to monotonically decrease and increase respectively with hole density, as shown in Fig. 4. Because the band becomes very narrow as the hole density decreases the chemical potential can fall below the bottom of the band giving rise to behavior very different to "conventional BCS", in particular the gap will not go to zero as $\mathrm{T} \rightarrow \mathrm{T}_{\mathrm{c}}{ }^{26}$

We are aware of a single report on measurements of $\mathrm{H}_{\mathrm{c} 2}$ versus hole concentration, 37 which shows monotonically decreasing behavior in agreement with the theory. More experiments are highly desirable. The monotonic decrease in normal state effective mass predicted should be evident in a variety of normal state properties but we have not perfomed a detailed analysis. Unusual behavior of the gap versus temperature has been reported in various experiments, 38 in particular tunneling.

\section{(6) Other properties}

Unusual behavior in normal state properties can result from a very narrow band, as we expect in this theory for low hole concentration. This is discussed for example by Scalapino et al.39 and Ashkenazi et al.40 Various other observables in the superconducting state like spin susceptibility, ultrasonic attenuation, NMR relaxation rate and frequency dependent conductivity follow behavior very similar to 
"conventional BCS", except for effects arising in the low density regime from the extreme narrowness of the band. 25,41 The observed absence of NMR relaxation peak appears to contradict the theory but could be related to a variety of effects, and it should be kept in mind that such a peak has often not been observed in "conventional" type II superconductors. 42

\section{(7) Effect of disorder}

The effect of impurity scattering on the critical temperature in this theory has recently been studied by Marsiglio. $43 \mathrm{He}$ finds that non-magnetic impurity scattering can have an important pair-breaking effect due to the energy dependance of the gap function Eq. (24). In the low-doping strong-coupling regime non-magnetic and magnetic impurities cause a reduction in $\mathrm{T}_{\mathrm{c}}$ of comparable magnitude. This finding is in qualitative agreement with observations on the effect of various experiments where $\mathrm{Cu}$ ions in the plane are replaced by other transition-metal ions. 44

\section{(8) Multi-band effects}

In the oxide superconductors we envisage superconductivity as arising from pairing of hole carriers that conduct through direct hopping between 0 anions, i.e. in a band formed by $0 \mathrm{p} \pi$ orbitals in the plane. This is in contradiction with the more commonly held view that doped holes go into $0 \mathrm{p} \sigma$ orbitals, that appears to be supported by certain experiments. 45 We believe that further analysis of these experiments will prove this view wrong.

Nevertheless, in the metallic regime conduction will occur also through the band formed by $0 \mathrm{p} \sigma-\mathrm{Cud}_{\mathrm{x}^{2}-\mathrm{y}^{2}}$ orbitals, and while these carriers do not play a central role in the superconductivity they can give rise to observable effects. To describe this situation we have recently studied a simple two-band model with holelike and electron-like carriers. 46 One of the effects of the second band is to give rise to a second, smaller, superconducting gap associated with the (weaker) binding of the electron carriers, and as a consequence additional structure in tunneling characteristics, that resembles experimental observations. 47 Another more fundamental effect that is specific to the model discussed here is the expected behavior of the Hall resistivity near the critical temperature: as the magnetic field is increased at temperatures somewhat below $\mathrm{T}_{\mathrm{c}}$ the model predicts the Hall resistivity to turn negative first as the weakly bound electron pairs are driven normal, and to turn positive at larger fields as the hole carrier pairs also break up. Such behavior is observed experimentally in a variety of samples. 48

\section{DISCUSSION}

The theory discussed above rests on the premise that electron-hole asymmetry is a fundamental feature of solids. This premise is in contradiction with commonly held views 49 but is clearly not demonstrably wrong. Its fundamental origin is to be sought in the physics of the atoms that form the solids and is clearly demonstrated by the simple fact that the periodic table manifestly does not exhibit left-right symmetry across its center.

Starting from this premise, and with the single assumption that holes are heavier than electrons, a theory of superconductivity results that in its simplest form exhibits a remarkable number of features observed in the high $\mathrm{T}_{\mathrm{c}}$ oxides. The theory makes in addition a number of detailed predictions that should be experimentally verifiable in the near future. Furthermore, while these materials display most clearly the physics that leads to superconductivity, they are by no means qualitatively different from other superconductors; similarly the theory discussed here naturally extends to all superconductors and thus offers the possibility of a unified explanation for superconductivity in all solids.

The electron-phonon mechanism is the accepted origin of superconductivity in "conventional" materials 50 . However, for many observations supposedly understood within 
that mechanism, the mechanism discussed here provides alternative natural explanations. 11 Isotope effect and phonon structure in tunneling, conventionally understood from phonon modulation of the hopping $t$, would be expected in our model from a (weaker) phonon modulation of $\Delta t$. The propensity of superconductors to lattice instabilities, and their large normal state resistivity, are conventionally understood as arising from large electron-phonon coupling; in our theory they are naturally expected from the fact that antibonding states are occupied in superconductors and from the large hole effective mass respectively. For many other observations like Matthias's rules ${ }^{51}$, Chapnik's rule and the existance of high $\mathrm{T}_{\mathrm{c}}$ oxides the mechanism discussed here has a natural explanation and the electron-phonon mechanism does not.

So does the electron-phonon interaction or the hole nature of the carriers lead to superconductivity in "conventional" materials? Given any superconductor, the theory discussed here suggests that if its ionic mass is increased to infinity without altering other properties the superconducting $\mathrm{T}_{\mathrm{c}}$ would remain finite, while if instead its Fermi surface is altered to eliminate all regions of negative curvature $T_{c}$ would go to zero. The electron-phonon mechanism would suggest the opposite behavior. Until experimentalists succeed in performing these "thought experiments" the question may not be strictly speaking settled; however, we suggest that it is already answered by the examples of $\mathrm{Pb}$ and Li respectively, and more generally by the systematics displayed by superconducting elements and compounds that show absolutely no correlation with the magnitude of the ionic mass but a strong correlation with the electron/holelike nature of the carriers.

In my view the fact that from a very simple principle such a large number of consequences emerge that are in agreement with observations represents compelling evidence that the principle is correct and the resulting theory describes reality. It would be indeed remarkable if nature had failed to take advantage of such a simple way to achieve superconductivity in solids while at the same time exhibiting so many features that point towards this explanation.

Acknowledgements: I am grateful to F. Marsiglio for collaboration in this work, to the National Science Foundation for support under NSF-DMR-8918306, to J. Torrance for permission to reproduce Fig. 1, and to the organizers of this meeting and particularly to J. Ashkenazi for their kindness and hospitality.

\section{$\underline{\text { References }}$}

1. I.M. Chapnik, Sov. Phys. Doklady ㅁ, 988 (1962); Phys. Lett. 72A, 255 (1979); J. Phys. F 13, 975 (1983).

2. C.M. Hurd, "The Hall Effect in Metals and Alloys", Plenum, New York, 1972, Chpt. 3.

3. I am told for example that B. Matthias was well aware of this rule (D. Wohlleben, private communication).

4. S. Uchida et al., Jpn. J. Appl. Phys. 26, 440 (1987); H. Takagi et al., in "Mechanisms of High Temperature Superconductivity," ed. by H. Kamimura and A. Oshiyama, Springer, Berlin, 1989, p. 238 and references therein.

5. Y. Tokura, H. Takagi and S. Uchida, Nature 337, 345 (1989). A large number of theoretical and experimental papers followed emphasizing that electron-hole symmetry in oxides was now an established fact. See for example the commentary by R. Pool in Science 243, 1436 (1989).

6 J. E. Hirsch, "Electron Superconductivity in Oxides?", UCSD Report, March 2, 1989. (Submitted to Nature and Science correspondance sections, journals declined publication.)

7. J. E. Hirsch and F. Marsiglio, Phys. Lett. A 140, 122 (1989).

8. N. Nucker et al., Z. Phys. B 75, 421 (1989).

9. J. M. Tarascon, private communication, July 1989; Y. Tokura et al., reported at the APS March meeting, 1990; Y. Iye et al., reported at APS March meeting, 1990.

10. J. B. Torrance et al., Phys. Rev. Lett. 61, 1127 (1988); Phys. Rev. B40, 8872 (1989).

11. J. E. Hirsch, Physica C 158, 326 (1989); Phys. Lett. A138, 83 (1989); Mat. Res. Soc. Symp. Proc. Vol. 156, 349 (1989).

12. J E. Hirsch, Phys. Rev. Lett. 59, 228 (1987); Y. Takashashi, Z. Phys. B 67,503 (1987). 
13. T. Izuyama, Physica B 169,995 (1990), and references therein.

14. R. B. Laughlin, in "Mechanisms of High Temperature Superconductivity," ed. by H. Kamimura and A. Oshiyama, Springer, Berlin, 1989, P. 76.

15. J. E. Hirsch, Physica C 161, 185 (1989).

16. M. Takigawa et al., Phys. Rev. B 39, 7371 (1989).

17. J. E. Hirsch, Phys. Lett. A 134, 451 (1989); J. E. Hirsch and S. Tang, Phys. Rev. B 40 , 2179 (1989); Sol. St. Comm. $\underline{69}, 987$ (1989); J. E. Hirsch and F. Marsiglio, Phys. Rev. B 41, 2049 (1990).

18. J. E. Hirsch, Phys. Rev. B (in press).

19. M. S. Hybertsen et al., Phys Rev. B 41,11068 (1990).

20. J. E. Hirsch, Chem. Phys. Lett. 171, 161 (1990).

21. J. Zaanen, C. Westra and G. Sawatzky, Phys. Rev. B33, 8060 (1986).

22. O. Gunnarson and N. E. Christensen, Phys. Rev. B42, 2363 (1990) and references therein.

23. A. Zawadowski, Phys. Scripta VT27, 66 (1989) and references therein.

24. J. E. Hirsch and F. Marsiglio, Phys. Rev. B $\underline{39}, 11515$ (1989); Physica C 162-164, 591 (1989).

25. F. Marsiglio and J. E. Hirsch, Phys. Rev. B $\underline{41}, 6435$ (1990); Physica C 162-164, 1451 (1989).

26. F. Marsiglio and J. E. Hirsch, Physica C 165, 71 (1990); Physica C 171, 554 (1990).

27. F. Marsiglio and J. E. Hirsch, Physica C 159,157 (1989).

28. N. N. Bogoliubov, Nuovo Cimento I, 794 (1958); P. Morel and P. W. Anderson, Phys. Rev. 125, 1263 (162).

29. R. Micnas, J. Ranninger and S. Robaskiewicz, Rev. Mod. Phys. 62, 113 (1990).

30. A. J. Legget, J. Phys. (Paris) 41, C7 (1980); P. Nozieres and S. Schmitt-Rink, J. Low Temp. Phys. 59, 195 (1985).

31. R. Micnas, J. Ranninger and S. Robaskiewicz, Phys. Rev. B $\underline{39}, 11653$ (1989).

32. C. G. Olson et al., Sol. St. Comm. 76, 411 (1990).

33. M. F. Crommie et al., Phys. Rev. B $\underline{39}, 4231$ (1989).

34. C. Meingast et al., Phys. Rev. B 41, 11299 (1990).

35. A. Gupta et al. Phys. Rev. Lett. 64, 3191 (1990).

36. J. E. Hirsch and F. Marsiglio, Physica C 172, 265 (1990).

37. M. Suzuki and M. Hikita, Jap. Jour. Appl. Phys. 28, L1368 (1989).

38. See various contributions in these proceedings.

39. D. J. Scalapino, R. T. Scalettar and N. E. Bickers, in "Novel Superconductivity", ed. by S. E. Wolf and V. Z. Kresin, Plenum, New York, 1987, p.475.

40. J. Ashkenazi et al., these proceedings.

41. F. Marsiglio and J. E. Hirsch, unpublished.

42. B. G. Silbernagel et al., Phys. Rev. 153, 535 (1967); W. Fite, II, and A. G. Redfield, Phys. Rev. Lett. 17, 381 (1966)).

43. F. Marsiglio, unpublished.

44. J. M. Tarascon et al., Phys. Rev. 337, 7458 (1988).

45. F. J. Adrian, Physica C $171,505(1990)$ and references therein.

46. J. E. Hirsch and F. Marsiglio, Phys. Rev. B43, 424 (1991).

47. M. Gurvitch et al, Phys. Rev. Lett. 63, 1008 (1989).

48. Y. Iye, S. Nakamura and T. Tamegai, Physica C $\underline{159}$, 616 (1989); S. J. Hagen et al., Phys. Rev. B 41, 11630 (1990).

49. W. Heisenberg, Ann. der Phys. 10, 883 (1931); N. W. Ashcroft and N. D. Mermin, "Solid State Physics", Holt, Reinhart and Winston, New York, 1976, Chpt. 12.

50. See for example various chapters in "the last nail in the coffin of superconductivity": "Superconductivity", ed. by R. D. Parks, Marcel Dekker, New York, 1969.

51. B. T. Matthias, Prog. in Low Temp. Phys. Vol. 2, ed. by J. C. Gorter, North Holland, Amsterdam, 1957, p. 138. 